\title{
Non-medical costs of colonoscopy
}

\author{
Paweł W. Petryszyn ${ }^{1}$, Radosław Kempiński ${ }^{2}$, Jerzy Michałowicz ${ }^{3}$, Elżbieta Poniewierka ${ }^{4}$ \\ ${ }^{1}$ Department of Clinical Pharmacology, Wroclaw Medical University, Wroclaw, Poland \\ ${ }^{2}$ Department of Gastroenterology and Hepatology, Wroclaw Medical University, Wroclaw, Poland \\ ${ }^{3}$ Department of Internal Medicine and Gastroenterology, Regional Hospital, Kalisz, Poland \\ ${ }^{4}$ Division of Dietetics, Department of Gastroenterology, Wroclaw Medical University, Wroclaw, Poland
}

Prz Gastroenterol 2014; 9 (5): 270-274

DOI: $10.5114 / p g .2014 .46161$

Key words: cost analysis, colonoscopy.

Address for correspondence: Paweł W. Petryszyn MD, Department of Clinical Pharmacology, Wroclaw University of Medicine, 213 Borowska St, 50-571 Wroctaw, Poland, phone: +48 693337 296, fax: +48 7173321 29, e-mail: ppetryszyn@wp.pl

\begin{abstract}
Colorectal cancer is one of the most common malignancies in Europe and North America. Colonoscopy done every 10 years beginning at age 50 is the preferred method of screening. In Poland and some other countries examinations are offered to subjects free of charge. However, as well as direct medical costs there are direct non-medical costs, which include the cost of transportation and costs related to caregivers' time, and indirect costs, which are costs related to patients' time. These costs essentially augment the total societal costs of colonoscopy.
\end{abstract}

\section{Introduction}

Colorectal cancer is one of the most common malignancies in Europe and North America. It is the second leading cause of cancer death in men and women, exceeded only by lung cancer $[1,2]$. Because of its high prevalence, long asymptomatic phase, and the presence of treatable precancerous lesions, colorectal cancer ideally fulfils the World Health Organisation criteria for population screening [3]. The majority of colorectal cancers arise through the following sequence: normal colon - non-advanced adenoma - advanced adenoma - colorectal cancer. Patients with colorectal cancer found while screening have improved 5-year survival compared with patients presenting with symptomatic cancer. It is caused by more favourable stage distribution at the time of diagnosis - cancers found through screening are generally in their earlier stages $[4,5]$. In many countries scientific associations recommend that average-risk individuals begin screening for colorectal cancer at age 50. The following screening modalities are available: faecal occult blood test, faecal immunochemical tests, detection of DNA shed by neoplastic tissues in stool, double-contrast barium enema, computed tomographic colonography or virtual colonoscopy, flexible sigmoidoscopy, or colonoscopy. Faecal occult blood tests are most often guaiac-based. The main arguments in favour of colonoscopy against FOBTs are as follows: FOBTs need to be repeated annually, while colonoscopy is offered every 10 years; the sensitivity of a single FOBT for identifying colorectal cancer, and in particular adenomas, is low, whereas colonoscopy has a rather high sensitivity (i.e. $85 \%$ for non-advanced adenoma, $87.5 \%$ for advanced adenoma, and $96.6 \%$ for colorectal cancer); and colonoscopy permits the removal of precancerous lesions (i.e. polyps-adenomas during a screening examination) $[4,6]$. According to American College of Gastroenterology guidelines, the colorectal cancer screening tests are grouped into cancer prevention tests (allowing the visualisation of both cancer and polyps) and cancer detection tests (having low sensitivity for identifying polyps and inferior sensitivity compared to cancer prevention tests in identifying neoplasia). The cancer prevention tests are recommended. Colonoscopy every 10 years beginning at age 50 remains the preferred colorectal cancer screening strategy. Alternative colorectal cancer prevention tests are flexible sigmoidoscopy every 5-10 years and computed tomographic colonography every 5 years. In patients in whom cancer prevention tests cannot be performed for some reason, the preferred cancer detection test is annual faecal immunochemical test. Alternatives are FOBT annually and faecal DNA test every 3 years [7]. Colorectal cancer 
screening is widely accepted and funded in European Union countries; nowadays adequate programs exist in 19 out of 27 member countries. In Poland in 2000 the National Colorectal Cancer Screening Program was launched, eligible for people 50-65 years of age who are asymptomatic and people 40-65 years of age having a family history of cancer of any type and based on a single colonoscopy [6].

The aim of the study is to evaluate direct non-medical costs and indirect costs of colonoscopy in the colorectal cancer screening method, based on the literature review.

\section{Cost types}

A cost in pharmacoecomic analysis is considered as the amount of expenditure that has been used to carry out a given health program in consequence of which a certain outcome was achieved. The costs imposed on society as a result of a medical condition or a clinical procedure are divided into the following:

- direct medical costs, i.e. costs embracing all resources employed by health care providers during medical treatment, e.g. costs of diagnostic procedures, drug acquisition costs, costs of monitoring therapy, costs of adverse events management, hospitalisation costs, medical staff costs, costs of specialist consultations, and administrative costs;

- direct non-medical costs, i.e. the value of nonmedical goods, services, and other resources that are consumed with respect to a condition or procedure, such as costs of a special diet or transportation to and from a treatment centre;

- indirect costs denote productivity losses due to incapacity for work, reduced work productivity, or loss of leisure time;

- and intangible costs, i.e. related to pain, worry, and other distress a patient or their family might suffer.

The entire process of cost estimation is determined by the chosen study perspective. If we decide on the perspective of the health-care payer, indirect costs and direct costs not reimbursed by the health-care payer are not taken into account. If the societal perspective is chosen, all costs are included regardless of who incurs them. In general the societal perspective, which is the broadest one, is considered the most appropriate as the aim of pharmacoeconomic analysis is the best possible resource allocation and the society as whole provides public money. Evaluation using this perspective, which entails identification and consideration of all the above three cost types: direct medical costs, direct non-medical costs, and indirect costs, avoids distortions otherwise induced by cost shifting. Intangible costs are impossible to measure in monetary terms and are not considered in pharmacoeconomic analysis or alternatively captured in measures of quality of life. If we were to consider resource use within a narrower perspective, for example that of the patient or the health-care payer, then the more the patient or the public payer could pass costs on to other agents, the more cost effective would that intervention appear to be, from their own, mainly private perspective. Likewise, the intervention would appear cost ineffective to the other agents. Therefore, the evaluation from the societal perspective constitutes a playing field, where interventions involving costs incurred by different agents in varying proportions are compared [8,9]. Direct non-medical and indirect costs may have an impact on patients' access to some health-care services, especially in the field of prevention, such as screening. The use of health care services is inversely correlated with the price paid or costs incurred: higher prices or costs incurred tend to deter purchase or use, whereas lower prices encourage greater adherence [10]. Given that the effectiveness of programmes of mass screening depends primarily upon subjects' participation or compliance, it is of great importance that the price patients are charged is low. In the United Kingdom screening for cervical and breast cancer is routinely offered free of charge, and primary care physicians are given financial incentives to encourage recruitment to the programme [11]. It is important to bear in mind that even if the population screening is offered free within the health-care system, subjects are likely to incur extra costs. Most patients will incur expenses on travel and transport to the centre where the screening examinations are carried out. These are not reimbursable from public funds. Additionally, subjects will incur the opportunity costs of the time they spend in travelling and attending the procedure. Opportunity cost refers to the value of the next best alternative forgone. Participation in screening programmes may, but does not have to, result in a direct loss of income, depending on the terms and conditions of employment. Potential screening users perceiving their time as valuable or travel costs as high may withhold attendance for screening even though they are offered free $[8,9]$.

\section{Time and travel costs}

In the screening programmes based on regular testing for the presence of faecal occult blood, time and travel costs are almost negligible. Patients receive the test by mail, complete it in their own home, and then return it via pre-paid postage to a centre for processing. In contrast, colonoscopy necessitates a clinic visit [12]. Travel costs were analysed in the studies by Frew et al. and Henry et al. The former was a survey study and comprised 3525 respondents from 12 clinical centres in 
Great Britain that had carried out flexible sigmoidoscopy as screening for colorectal cancer. Flexible sigmoidoscopy does not allow complete colonic evaluation and is undoubtedly less expensive in comparison with colonoscopy; however, it may be assumed that both examinations do not differ as far as direct non-medical and indirect costs are concerned. $80.5 \%$ respondents travelled to the clinic by car, $9.2 \%$ by bus, $3.5 \%$ by taxi, $1.5 \%$ by train, $1.4 \%$ walked, and the remaining $3.9 \%$ came by other means or a combination of modes. The starting point for the majority of subjects (96.4\%) was their own home. Of this group, $92.7 \%$ subsequently returned home and 5.2\% went to work. The mode of transport distribution was dependent on the centre: in Norwich and Portsmouth there was the largest proportion of car users (99.2\% and 95.5\%, respectively), in Leeds the largest proportion of bus users (32.2\%), in Glasgow the largest proportion of taxi users (13.2\%), and London recorded the highest proportion of rail travellers (10.8\%). Car travel costs were calculated by multiplying travel miles by the applicable rate per mile for the kind of vehicle being used. An average estimate of 40 pence a mile for 1100-2000 cc vehicles travelling 10 000-15 000 miles a year was used. Mean round trip time was $56 \mathrm{~min}$ and mean round trip distance was 14.4 miles $(22.8 \mathrm{~km})$. Mean travel times for car and taxi users were significantly shorter than travel times for bus users and other modes combined. The mean distance travelled by car users was significantly higher than that travelled by all other transport mode users. Transport costs varied significantly in respect to the transport mode, being the highest for taxi users and the lowest for bus travellers. The mean travel cost amounted to $\$ 6.10$ per person. About 2/3 of respondents were accompanied by other people. Most often the accompanying person was their partner (57.4\%). Women, housewives, retired, and car and taxi users were significantly more often accompanied during the clinic visit. Of those attending alone, $38.4 \%$ indicated that they would have been working if they had not been attending the clinic and $60.7 \%$ would have been engaged in unpaid activities like leisure or housework. $22.4 \%$ of companions lost working hours; for 1 in 10 of the accompanied visits both the subject and companion lost working hours and in $37.9 \%$ of visits at least one of them lost working hours. Mean total time and travel cost was estimated for a subject as $\$ 16.90$ and for a screening attendance as $\$ 22.40$. Lost work time costs were based on average hourly rate of pay depending on sex, manual vs. non-manual occupations, and country region. Direct non-medical and indirect costs of flexible sigmoidoscopy screening were in the same range as the direct medical costs of the procedure itself, doubling its total cost to the society. The authors also compared the distribution of socioeconomic classes within flexible sigmoidoscopy screening participants with that of the population of Great Britain as a whole and drew two conclusions: relative to the whole population, non-manual classes were more strongly represented in the flexible sigmoidoscopy sample $(60.1 \%$ vs. $51.3 \%)$, whereas the self employed were the least underrepresented. By attending clinic-based screening self-employed people lose earnings, which could effectively discourage them [9]. Henry et al. performed a cost analysis of colonoscopy using micro-costing and time-and-motion techniques. In the screening colonoscopy process several caregiving (i.e. involving direct provision of health care) and coordinating transactions were distinguished, and then the resource use, including the time each type of health care worker spent with each patient for all caregiving transactions, was assessed. Additionally, patients completed a questionnaire asking about distance travelled and cost incurred for travel, costs of child and elderly care, and time taken out of their or their drivers' usual schedule for the procedure. The median direct health care cost for colonoscopy was $\$ 379$, the median direct non-health care costs (travel costs and costs of caregivers' time) and indirect costs (related to patient time) were $\$ 226$ and $\$ 274$, respectively. Thus the median total societal cost of screening colonoscopy was $\$ 923$ [13]. Dong et al. contacted by telephone 68 patients who had colonoscopies performed for the indications of average risk screening or surveillance, e.g. in case of polypectomy. Other inclusion criteria were: age under 65 years, procedures scheduled for the middle of the week (excluding Mondays and Fridays), and being at workforce. Patients were asked about work time lost because of the procedure. $34 \%$ of patients took more than one day off from work, $32 \%$ took the day before the procedure off, primarily in anticipation of the bowel preparation, $10 \%$ took the day after the colonoscopy off, mainly as a precautionary measure after sedation rather than in response to true symptoms, and $9 \%$ of patients took both the day before and the day after off. Forty-six percent of patients had an accompanying person who had to miss work as a result of the colonoscopy procedure. Assuming $50 \%$ utilisation of screening colonoscopy, if $34 \%$ of US adults age 50-65 years took one additional day off from work, that would amount to $\$ 778$ million. This cost may be minimised through patient education about bowel preparation techniques and what to expect before and after the procedure, and by scheduling more screening colonoscopies on Mondays and Fridays [14]. Jonas et al. defined different time intervals in relation to screening colonoscopy: total time, from changing one's diet in preparation for the procedure until feeling 'back 
to normal' after the procedure, preparation to routine time, from taking the preparation medication until returning to routine activities, occupied time, from taking the preparation medication until arriving at home or other destination, and dedicated time, from leaving home to go to the screening centre until arriving at home or other destination after the procedure. One hundred and ten subjects participated in the study, respective mean time values were as follows: total time $-81.5 \mathrm{~h}$, preparation to routine time $-39.9 \mathrm{~h}$, occupied time $-23.2 \mathrm{~h}$, and dedicated time $-4.4 \mathrm{~h}$. The human capital method equates the value of human life to the discounted market value of the output produced by an individual over an expected lifetime. It allows the convertion of time to money. Using national average wage rates, the mean values of time measures were estimated as follows: total time $-\$ 1518$, preparation to routine time - \$743, occupied time - \$432, and dedicated time - \$81. Using subjects' personal income data to evaluate the time produced significantly larger mean values: total time - \$2419, preparation to routine time - \$1147, occupied time - \$702, and dedicated time - \$122. The time spent during colonoscopy preparation is an irregular series of trips to the bathroom with intermittent periods of normalcy and sleep. Recovery may follow a similar pattern. Patients may be able to multitask during the preparation, for example by reading while they are in the bathroom. In such situations evaluating time by the wage, as the human-capital method does, may overestimate both the amount of time and its value to the patient. The willingness-to-pay method seems to be more appropriate. It not only allows evaluation of the time actually devoted to the process but also of the discomfort associated with the procedure. Patients were asked the maximum amount they were willing to pay to avoid the preparation, discomfort, and recovery period associated with the colonoscopy process while still receiving its benefits. On average it was $\$ 263$ and was substantially lower than the human capital values for the three longest time intervals but greater than the human capital values for dedicated time. Linear regression analysis showed that willingness-to-pay values were related to the difficulty of the preparation [15].

\section{Potential implications}

In the studies cited above, direct non-medical costs and indirect costs constituted together $50-60 \%$ total societal costs of the colonoscopy. Considering colonoscopy costs from the perspective of the whole of society is further supported by the fact that the National Colorectal Cancer Screening Program, which has been functioning in Poland since 2000, is funded by the Ministry of Health instead of the National Health Fund.
Therefore, cost effectiveness and cost utility analyses in this domain should rather be done from the societal perspective. The evidence that direct medical costs making up less than half of its total cost will have an influence on the result of incremental analysis comparing different colorectal cancer screening methods. High direct non-medical costs and caregivers' time and indirect costs (costs of patients' time) of the colonoscopy, if taken into account, may result in relatively higher cost effectiveness/utility ratio of colonoscopy once every 10 years against FOBT annually. Test repetition has a negative impact on how many persons remain in the screening programme, which means higher cost per lifeyear saved. The magnitude of this effect is obviously most pronounced in the case of FOBT, which has to be repeated annually. Sensitivity analysis has shown that a decrease of compliance to $90 \%$ increases the incremental cost effectiveness ratio (ICER) of faecal occult blood testing in comparison with no screening from $\$ 9705$ to $\$ 14788$. The same incremental cost effectiveness ratio is achieved with a decrease in compliance with repeated 10-year colonoscopy to 66\% (\$10983 at baseline). Similarly, a decrease in compliance with repeated FOBT to $80 \%$ matches a decrease in compliance with repeated colonoscopy to $37 \%$ [16]. However, high direct non-health-care and patient time costs may impact colonoscopy-based screening rates, yet thought to be one of its strongest points.

\section{References}

1. Siegel R, Naishadham D, Jemal A. Cancer statistics, 2012. CA Cancer J Clin 2012; 62: 10.

2. Bosetti C, Levi F, Rosato V, et al. Recent trends in colorectal cancer mortality in Europe. Int J Cancer 2011; 129: 180-91.

3. Wilson JMG, Jungner G. Principles and practice of screening for disease. WHO Chronicle Geneva: World Health Organization 1968; 22: 473.

4. Heitman SJ, Hilsden RJ, Au F, et al. Colorectal cancer screening for average-risk North Americans: an economic evaluation. PLoS Med 2010; 7: e1000370.

5. Mandel JS, Bond JH, Church TR, et al. Reducing mortality from colorectal cancer by screening for fecal occult blood. Minnesota Colon Cancer Control Study. N Engl J Med 1993; 328: 1365-71.

6. Reguła J. Screening in early colorectal cancer detecting. In: Basis of clinical oncology [Polish]. Meder J (ed.). CMKP, Warsaw 2011; 25-8.

7. Rex DK, Johnson DA, Anderson JC, et al. American College of Gastroenterology guidelines for colorectal cancer screening 2009 [corrected]. Am J Gastroenterol 2009; 104: 739.

8. Nowakowska E, Orlewska E. Definition of costs and outcomes in pharmacoeconomics. In: Pharmacoeconomics [Polish]. Akademia Medyczna im. Karola Marcinkowskiego, Poznan 2004; 12-25. 
9. Frew E, Wolstenholme JL, Atkin W, Whynes DK. Estimating time and travel costs incurred in clinic based screening: flexible sigmoidoscopy screening for colorectal cancer. J Med Screen 1999; 6: 119-23.

10. Giuffrida A, Torgerson DJ. Should we pay the patient? Review of financial incentives to enhance patient compliance. BMJ 1997; 315: 703-7.

11. Whynes DK, Baines DL. Income-based incentives in UK general practice. Health Policy 1998; 43: 15-31.

12. Walker A, Whynes DK, Chamberlain JO, Hardcastle JD. The cost of screening for colorectal cancer. J Epidemiol Community Health 1991; 45: 220-4.

13. Henry SG, Ness RM, Stiles RA, et al. A cost analysis of colonoscopy using microcosting and time-and-motion techniques. J Gen Intern Med 2007; 22: 1415-21.

14. Dong MH, Kalmaz D, Savides TJ. Missed work related to midweek screening colonoscopy. Dig Dis Sci 2011; 56: 2114-9.

15. Jonas DE, Russell LB, Chou J, Pignone M. Willingness-to-pay to avoid the time spent and discomfort associated with screening colonoscopy. Health Econ 2010; 19: 1193-211.

16. Sonnenberg A, Delcò F, Inadomi JM. Cost-effectiveness of colonoscopy in screening for colorectal cancer. Ann Intern Med 2000; 133: 573-84.

Received: 23.05 .2012

Accepted: 4.04 .2013 